\title{
A SPECIAL CASE OF THE BUCHSBAUM-EISENBUD-HORROCKS RANK CONJECTURE
}

\author{
DANIEL ERMAN
}

\begin{abstract}
The Buchsbaum-Eisenbud-Horrocks rank conjecture proposes lower bounds for the Betti numbers of a graded module $M$ based on the codimension of $M$. We prove a special case of this conjecture via Boij-Söderberg theory. More specifically, we show that the conjecture holds for graded modules where the regularity of $M$ is small relative to the minimal degree of a first syzygy of $M$. Our approach also yields an asymptotic lower bound for the Betti numbers of powers of an ideal generated in a single degree.
\end{abstract}

\section{Introduction}

Let $k$ be any field, let $S=k\left[x_{1}, \ldots, x_{n}\right]$ be the polynomial ring with the usual grading, and let $M$ be a graded $S$-module. The Buchsbaum-Eisenbud-Horrocks rank conjecture (herein the BEH rank conjecture) says roughly that the Koszul complex is the "smallest" possible minimal free resolution. ${ }^{1}$ The conjecture was formulated by Buchsbaum and Eisenbud in [3, p. 453] and, independently, the conjecture is implicit in a question of Horrocks [15, Problem 24]. Although the conjecture is most commonly phrased for regular local rings, we consider the graded case. Let

$$
0 \longrightarrow F_{p} \stackrel{\phi_{p}}{\longrightarrow} F_{p-1} \stackrel{\phi_{p-1}}{\longrightarrow} \cdots \stackrel{\phi_{1}}{\longrightarrow} F_{0} \stackrel{\phi_{0}}{\longrightarrow} M \longrightarrow 0
$$

be the graded minimal free resolution of $M$. Let $\beta_{j}(M):=\operatorname{rank}\left(F_{j}\right)$.

Conjecture 1.1 (Graded BEH Rank Conjecture). Let $M$ be a graded Cohen-Macaulay S-module of codimension c. Then:

$$
\beta_{j}(M) \geq\left(\begin{array}{l}
c \\
j
\end{array}\right)
$$

for $j=0, \ldots, c$.

In this paper, we prove a special case of the graded BEH rank conjecture. We do not require that $M$ is Cohen-Macaulay.

Theorem 1.2. Let $M$ be a graded $S$-module of codimension $c$, generated in degree $\leq$ 0 , and let $\underline{d}_{1}(M)$ be the minimal degree of a first syzygy of $M$. If $\operatorname{reg}(M) \leq 2 \underline{d}_{1}(M)-2$, then

$$
\beta_{j}(M) \geq \beta_{0}(M)\left(\begin{array}{l}
c \\
j
\end{array}\right) .
$$

Received by the editors April 23, 2009.

2000 Mathematics Subject Classification. Primary 13D02; Secondary 13D25.

The author is partially supported by an NDSEG fellowship.

${ }^{1}$ Terminology for this conjecture is inconsistent in the literature. In some places this conjecture is known as Horrocks' Conjecture or as the Syzygy Conjecture. 


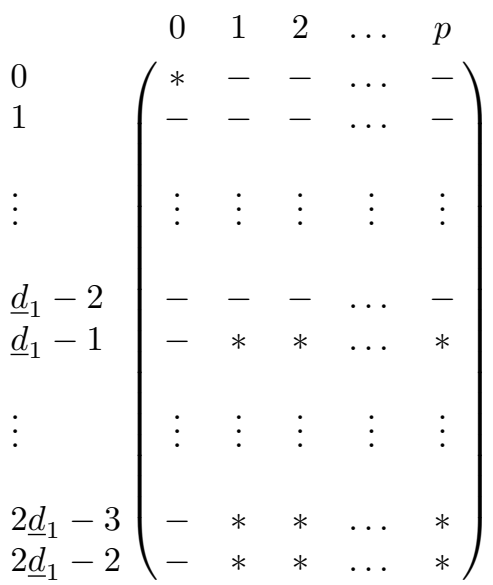

Figure 1. When $M$ has a Betti diagram of the above shape, then it satisfies the Buchsbaum-Eisenbud-Horrocks Rank Conjecture.

for $j=0, \ldots, c$.

Common generalizations of the $\mathrm{BEH}$ rank conjecture include removing the CohenMacaulay hypothesis and/or strengthening the conclusion to the statement that $\operatorname{rank}\left(\phi_{j}\right) \geq\left(\begin{array}{c}c-1 \\ j-1\end{array}\right)$ for $j=1, \ldots, c-1$. A different generalization, suggested in $[4$, Conj II.8], replaces the Betti numbers of a free resolution by the homology ranks of a differential graded module.

The BEH rank conjecture has been shown to hold for all modules of codimension at most 4 [14, p. 267]. In codimension at least 5, however, the BEH rank conjecture has only been settled for families of modules with additional structure.

Theorem 1.2 applies to modules whose Castelnuovo-Mumford regularity is small relative to the degree of the first syzygies of $M$. Though the literature on special cases of the BEH rank conjecture is extensive, Theorem 1.2 moves in a new direction. The most similar result in the literature is perhaps [5, Thm. 0.1], which shows that the BEH rank conjecture holds when $M$ is a Cohen-Macaulay module annihilated by the square of the maximal ideal $\mathfrak{m}$. Other known cases of the BEH rank conjecture include multigraded modules [7, Thm. 3] and [20], cyclic modules in the linkage class of a complete intersection [18], cyclic quotients by monomial ideals [14, Cor 2.5], and several more [6], [8], [11], and [17]. See [9, pp. 25-27] for an expository account of some of this progress.

The method of proof for Theorem 1.2 is quite different from previous work on the BEH rank conjecture. Our proof is an application of Boij-Söderberg theory, by which we mean the results of [13] and [1]. At first glance, it might appear that BoijSöderberg theory would not apply to Conjecture 1.1: Boij-Söderberg theory is based on the principle of only considering Betti diagrams up to scalar multiple, whereas the $\mathrm{BEH}$ rank conjecture depends on the integral structure of Betti diagrams. However, if the Betti diagram of $M$ has shape as in Figure 1, then this imposes conditions on the pure diagrams which can appear in the Boij-Söderberg decomposition of $M$. 
This allows us to reduce the proof of Theorem 1.2 to a statement about the numerics of pure diagrams. We then use a multivariable calculus argument to degenerate the relevant pure diagrams to a Koszul complex.

Our analysis of the numerics of pure diagrams also leads to a proposition about the asymptotic behavior of the Betti numbers of $S / I^{t}$ where $I$ is an ideal generated in a single a degree $\delta$. Let $c=\operatorname{codim}(S / I)$ and let $b$ be the asymptotic regularity defect of $I$ (see $\S 5$ for a definition). We will show that

$$
\beta_{j}\left(S / I^{t}\right) \geq \frac{(b !)^{2} \delta^{c-1}}{(j-1+b) !(c-j+b) !} t^{c-1}+O\left(t^{c-2}\right)
$$

for all $t \gg 0$ and all $j \in\{1, \ldots, c\}$.

This paper is organized as follows. In $\S 2$ we review the relevant aspects of BoijSöderberg theory. In $\S 3$, we investigate the numerics of pure diagrams which satisfy the conditions of Theorem 1.2. This analysis of pure diagrams is the foundation for the proof of Theorem 1.2, which appears in $\S 4$. In $\S 5$, we prove Proposition 5.1 about asymptotic Betti numbers. In $\S 6$, we consider applications of Theorem 1.2.

\section{Review of Boij-Söderberg theory}

We say that a sequence $\mathbf{d}=\left(d_{0}, \ldots, d_{s}\right) \in \mathbb{Z}^{s+1}$ is a degree sequence if $d_{i}>d_{i-1}$ for all $i>0$. We use the notation $\operatorname{deg}\left(\mathbb{Z}^{s+1}\right)$ for the space of degree sequences of length $s+1$. Given two degree sequence $\mathbf{d}, \mathbf{d}^{\prime} \in \mathbb{Z}^{s+1}$, we say that $\mathbf{d} \geq \mathbf{d}^{\prime}$ if $d_{i} \geq d_{i}^{\prime}$ for $i=0, \ldots, s$. If $s \leq p$ and $\mathbf{d}=\left(d_{0}, \ldots, d_{p}\right)$ is a degree sequence, then we define $\tau_{s}(\mathbf{d})$ to be the truncated degree sequence $\tau_{s}(\mathbf{d}):=\left(d_{0}, \ldots, d_{s}\right)$.

Each degree sequence $\mathbf{d}$ defines a ray in the cone of Betti diagrams; there exists a unique point $\bar{\pi}(\mathbf{d})$ on this ray with $\beta_{0, d_{0}}(\bar{\pi}(\mathbf{d}))=1$ (c.f. [13, Thm 0.1]). The diagram $\bar{\pi}(\mathbf{d})$ is the normalized pure diagram of type $\mathbf{d}$.

Note that $\bar{\pi}(\mathbf{d})$ may have non-integral entries. For instance

$$
\bar{\pi}(0,1,2,4)=\left(\begin{array}{cccc}
1 & \frac{8}{3} & 2 & - \\
- & - & - & \frac{1}{3}
\end{array}\right) .
$$

Given any diagram $D$ we define $\beta_{i}(D):=\sum_{j} \beta_{i, j}(D)$.

Let $M$ be a graded $S$-module of codimension $c$ and projective dimension $p$. For $i=0, \ldots, p$ we define $\underline{d}_{i}:=\min \left\{j \mid \beta_{i, j}(M) \neq 0\right\}$ and $\bar{d}_{i}:=\max \left\{j \mid \beta_{i, j}(M) \neq 0\right\}$. We set $\underline{\mathbf{d}}:=\left(\underline{d}_{0}, \ldots, \underline{d}_{p}\right)$ and $\overline{\mathbf{d}}:=\left(\bar{d}_{0}, \ldots, \bar{d}_{p}\right)$. Boij-Söderberg theory shows that the Betti diagram of any graded $S$-module can be expressed as a positive rational sum of pure diagrams which correspond to degree sequences bounded by $\overline{\mathbf{d}}$ and $\underline{\mathbf{d}}$. The following theorem is weaker than the main results of Boij-Söderberg theory, but it will be sufficient for our purposes.

Theorem 2.1 (Eisenbud-Schreyer (2008), Boij-Söderberg (2008)). Let $M$ be a graded $S$-module of projective dimension $p$ and codimension $c$ and let $\overline{\mathbf{d}}$ and $\underline{\mathbf{d}}$ as above. The Betti diagram $\beta(M)$ can be expressed as a sum:

$$
\beta(M)=\sum_{c \leq s \leq p} \sum_{\substack{\mathbf{d} \in \operatorname{deg}\left(\mathbb{Z}^{s+1}\right) \\ \tau_{s}(\underline{\mathbf{d}}) \leq \mathbf{d} \leq \tau_{s}(\overline{\mathbf{d}})}} a_{\mathbf{d}} \bar{\pi}(\mathbf{d})
$$

where each $a_{\mathbf{d}}$ is a nonnegative rational number. 
A stronger version of this theorem allows for a unique decomposition of $\beta(M)$ into a positive rational sum of pure diagrams and even provides an algorithm for producing this decomposition. See the introduction of [13] for an expository treatment of the main results of Boij-Söderberg theory, and see [13, §7] for a proof of of Theorem 2.1 in the case where $M$ is Cohen-Macaulay. See [1, Thm 2] for a proof of Theorem 2.1 when $M$ is not necessarily Cohen-Macaulay.

\section{Ranks of Pure Diagrams}

In this section, we study the numerics of the pure diagrams which satisfy the conditions of Theorem 1.2. Given any degree sequence $\mathbf{d}$, the Betti numbers of $\bar{\pi}(\mathbf{d})$ can be expressed as a rational function in the $d_{i}$, and we will use these rational functions to investigate the pure diagrams.

We introduce auxiliary functions to simplify the notation. For $\mathbf{e}=\left(e_{1}, \ldots, e_{s}\right) \in$ $\mathbb{R}^{s}$, we define linear functions, $T_{i}, U_{i, j}$, and $V_{i, j}$ from $\mathbb{R}^{s}$ to $\mathbb{R}$ by the following formulas:

$$
\begin{aligned}
T_{i}(\mathbf{e}) & :=i+e_{1}+e_{2}+\cdots+e_{i}, \text { for } i=1, \ldots, s \\
U_{i, j}(\mathbf{e}) & :=(j-i+1)+e_{i}+e_{i+1}+\ldots e_{j}, \text { whenever } i<j \\
V_{i, j}(\mathbf{e}) & :=(i-j)+e_{j+1}+\cdots+e_{i}, \text { whenever } i>j
\end{aligned}
$$

Let $\mathfrak{d}: \mathbb{R}^{s} \rightarrow \mathbb{R}^{s+1}$ be the linear map:

$$
\mathfrak{d}_{j}(\mathbf{e})= \begin{cases}0 & \text { for } j=0 \\ j+\sum_{i=0}^{j} e_{i}, & \text { for } j=1, \ldots, s .\end{cases}
$$

Note that $\mathbf{e} \in \mathbb{Z}_{\geq 0}^{s}$ if and only if $\mathfrak{d}(\mathbf{e})$ is a degree sequence with first entry equal to 0 .

We define the rational function $\mathfrak{b}_{j}: \mathbb{R}^{s} \rightarrow \mathbb{R}$ by:

$$
\mathfrak{b}_{j}(\mathbf{e}):=\frac{\left(\prod_{i=1, i \neq j}^{s} T_{i}\right)}{\left(\prod_{i=2}^{j-1} U_{i, j}\right)\left(\prod_{i=j+1}^{s} V_{i, j}\right)}
$$

for $j=1, \ldots, s$. The rational function $\mathfrak{b}_{j}$ has no poles on $\mathbb{R}_{\geq 0}^{s}$. The purpose of these definitions is summarized in the following lemma:

Lemma 3.1. Let $\mathbf{e} \in \mathbb{Z}_{\geq 0}^{s}$. Then we have:

$$
\mathfrak{b}_{j}(\mathbf{e})=\beta_{j}(\bar{\pi}(\mathfrak{d}(\mathbf{e})))
$$

Proof. For any degree sequence $\mathbf{d}$ of length $s$, a result of [16] can be used to give the explicit formulas

$$
\beta_{j}(\bar{\pi}(\mathbf{d}))=\prod_{\substack{1 \leq i \leq s \\ i \neq j}} \frac{\left|d_{i}-d_{0}\right|}{\left|d_{i}-d_{j}\right|}
$$

(c.f. [2, Defn 2.3]). Now let $\mathbf{d}=\mathfrak{d}(\mathbf{e})$ and fix some $i \neq j$. Observe that $\left|d_{i}-d_{0}\right|=T_{i}$; further, $\left|d_{i}-d_{j}\right|=U_{i, j}$ if $i<j$ and $\left|d_{i}-d_{j}\right|=V_{i, j}$ if $i>j$. This proves the lemma.

Lemma 3.2. On the domain $\mathbf{e} \in \mathbb{R}_{\geq 0}^{s}$, we have $\frac{\partial}{\partial e_{1}} \mathfrak{b}_{j} \geq 0$.

Proof. Consider the expression for $\mathfrak{b}_{j}$ given in (1), and observe that the denominator is not a function of $e_{1}$. Hence it is sufficient to show that $\frac{\partial}{\partial e_{1}}\left(\prod_{i=1, i \neq j}^{s} T_{i}\right) \geq 0$ when $\mathbf{e} \in \mathbb{R}_{\geq 0}^{s}$, and this is immediately verified. 
Lemma 3.3. Let $\mathbf{e} \in \mathbb{R}_{\geq 0}^{s}$ and fix some $j, k \in\{1, \ldots, s\}$.

(1) If $k<j$ then $\left(\frac{\partial}{\partial e_{j}}-\frac{\partial}{\partial e_{k}}\right) \mathfrak{b}_{j} \leq 0$.

(2) If $k>j+1$ then $\left(\frac{\partial}{\partial e_{j+1}}-\frac{\partial}{\partial e_{k}}\right) \mathfrak{b}_{j} \leq 0$.

Proof. Throughout this proof, we restrict all functions to the domain $\mathbf{e} \in \mathbb{R}_{\geq 0}^{s}$. It is sufficient to prove the statements for $\log \mathfrak{b}_{j}$. We may write:

$$
\log \mathfrak{b}_{j}=\sum_{\substack{1 \leq i \leq s \\ i \neq j}} \log T_{i}-\sum_{i=1}^{j-1} \log U_{i, j}-\sum_{i=j+1}^{s} \log V_{i, j}
$$

To prove part (1) of the lemma, we assume that $k<j$ and we fix some $i \in\{1, \ldots, s\}$ where $i \neq j$. Observe that

$$
\left(\frac{\partial}{\partial e_{j}}-\frac{\partial}{\partial e_{k}}\right) \log T_{i}=\left(\frac{\partial}{\partial e_{j}}-\frac{\partial}{\partial e_{k}}\right) \log \left(i+e_{1}+e_{2}+\cdots+e_{i}\right) \leq 0
$$

with equality if and only if $i<k$ or $i>j$. Similarly, if $i \in\{1, \ldots, j-1\}$, then

$$
\left(\frac{\partial}{\partial e_{j}}-\frac{\partial}{\partial e_{k}}\right) \log U_{i, j}=\left(\frac{\partial}{\partial e_{j}}-\frac{\partial}{\partial e_{k}}\right) \log \left(j-i+1+e_{i}+e_{i+1}+\cdots+e_{j}\right) \geq 0
$$

with equality if and only if $k<i$. Since $k<j$, we also have that

$$
\left(\frac{\partial}{\partial e_{j}}-\frac{\partial}{\partial e_{k}}\right) \log V_{i, j}=\left(\frac{\partial}{\partial e_{j}}-\frac{\partial}{\partial e_{k}}\right) \log \left(i-j+e_{j+1}+\cdots+e_{i}\right)=0
$$

for all $i \in\{j+1, \ldots, s\}$. By combining equation (2) with the results of these three computations, we conclude that $\left(\frac{\partial}{\partial e_{j}}-\frac{\partial}{\partial e_{k}}\right)\left(\log \mathfrak{b}_{j}\right) \leq 0$ as desired.

To prove part (2) of the lemma, we now assume that $k>j+1$ and we fix some $i \in\{1, \ldots, s\}$ with $i \neq j$. Observe first that $\left(\frac{\partial}{\partial e_{j+1}}-\frac{\partial}{\partial e_{k}}\right) \log U_{i, j}=0$ for all $i$; second, that if $i<j+1$ or $i \geq k$ then $\left(\frac{\partial}{\partial e_{j+1}}-\frac{\partial}{\partial e_{k}}\right) \log T_{i}=0$; and third, that if $i \geq k$ then $\left(\frac{\partial}{\partial e_{j+1}}-\frac{\partial}{\partial e_{k}}\right) \log V_{i, j}=0$. It remains to show that

$$
\left(\frac{\partial}{\partial e_{j+1}}-\frac{\partial}{\partial e_{k}}\right) \sum_{i=j+1}^{k-1} \log T_{i}-\log V_{i, j} \leq 0 .
$$

This follows from the computation: 


$$
\begin{array}{r}
\left(\frac{\partial}{\partial e_{j+1}}-\frac{\partial}{\partial e_{k}}\right) \sum_{i=j+1}^{k-1} \log T_{i}-\log V_{i, j}= \\
\sum_{i=j+1}^{k-1} \frac{1}{i+e_{1}+\cdots+e_{i}}-\frac{1}{i-j+e_{j+1}+\cdots+e_{i}} \\
=\frac{\left(i-j+e_{j+1}+\cdots+e_{i}\right)-\left(i+e_{1}+\cdots+e_{i}\right)}{\left(i+e_{1}+\cdots+e_{i}\right)\left(i-j+e_{j+1}+\cdots+e_{i}\right)} \\
=\frac{-j-e_{1}-\cdots-e_{j}}{\left(i+e_{1}+\cdots+e_{i}\right)\left(j-i+e_{j+1}+\cdots+e_{i}\right)}<0
\end{array}
$$

which completes the proof.

Lemma 3.4. Let $\mathbf{e} \in \mathbb{R}_{\geq 0}^{s}$ with $e_{1} \geq \sum_{j=1}^{s} e_{j}$. Then

$$
\mathfrak{b}_{j}(\mathbf{e}) \geq\left(\begin{array}{l}
s \\
j
\end{array}\right) .
$$

Proof. By Lemma 3.2, it is sufficient to prove the lemma in the case $e_{1}=\sum_{i=2}^{n} e_{i}$. Furthermore, by Lemma 3.3, we may assume that $e_{i}=0$ for $i \notin\{1, j, j+1\}$.

Assume for the moment that $j \notin\{1, s\}$ and let $\widetilde{\mathbf{e}}=\left(e_{1}, e_{j}, e_{j+1}\right)$. Under these assumptions we may write $\mathfrak{b}_{j}$ as a function of $\widetilde{\mathbf{e}}$. Our goal is to show that $\mathfrak{b}_{j}(\widetilde{\mathbf{e}}) \geq\left(\begin{array}{l}s \\ j\end{array}\right)$ given the constraint $e_{1}=e_{j}+e_{j+1}$ and the domain $\widetilde{\mathbf{e}} \in \mathbb{R}_{\geq 0}^{3}$.

We introduce a new variable $t$ and write $e_{j}=t e_{1}$ and $e_{j+1}=(1-t) e_{1}$. Under this change of coordinates, our constrained minimization problem is now equivalent to minimizing the function:

$$
\mathfrak{c}_{j}:=\frac{\left(1+e_{1}\right)\left(2+e_{1}\right) \cdots\left(j-1+e_{1}\right) \cdot\left(j+1+2 e_{1}\right) \cdots\left(n+2 e_{1}\right)}{\left(j-1+t e_{1}\right) \cdots\left(1+t e_{1}\right)\left(1+(1-t) e_{1}\right) \cdots\left((n-j)+(1-t) e_{1}\right)}
$$

over the domain $\left(t, e_{1}\right) \in[0,1] \times[0, \infty)$.

We claim that the minimum of $\log \mathfrak{c}_{j}$ on the domain $[0,1] \times[0, \infty)$ occurs when $e_{1}=0$. The partial derivative $\frac{\partial \log c_{j}}{\partial e_{1}}$ is the sum of the following 4 functions:

- $f_{1}:=\frac{1}{1+e_{1}}+\cdots+\frac{1}{j-1+e_{1}}$

- $f_{2}:=\frac{2}{j+1+2 e_{1}}+\cdots+\frac{2}{n+2 e_{1}}$

- $f_{3}:=-\frac{t}{1+t e_{1}}-\cdots-\frac{t}{j-1+t e_{1}}$

- $f_{4}:=-\frac{1-t}{1+(1-t) e_{1}}-\cdots-\frac{1-t}{(n-j)+(1-t) e_{1}}$ 
We observe first that:

$$
\begin{aligned}
-f_{1}-f_{3} & =\sum_{i=1}^{j-1} \frac{-1}{i+e_{1}}+\frac{t}{i+t e_{1}} \\
& =\sum_{i=1}^{j-1} \frac{-\left(i+t e_{1}\right)+t\left(i+e_{1}\right)}{\left(i+e_{1}\right)\left(i+t e_{1}\right)} \\
& =\sum_{i=1}^{j-1} \frac{(-1+t) i}{\left(i+e_{1}\right)\left(i+t e_{1}\right)} .
\end{aligned}
$$

Hence $-f_{1}-f_{3} \leq 0$ whenever $\left(t, e_{1}\right) \in[0,1] \times[0, \infty)$. We next observe that:

$$
\begin{aligned}
-f_{2}-f_{4} & =\sum_{i=j+1}^{n} \frac{-2}{i+2 e_{1}}+\frac{1-t}{i+(1-t) e_{1}} \\
& =\sum_{i=j+1}^{n} \frac{\left(-2 i-2(1-t) e_{1}\right)+\left((1-t) i+2(1-t) e_{1}\right)}{\left(i+2 e_{1}\right)\left(i+(1-t) e_{1}\right)} \\
& =\sum_{i=j+1}^{n} \frac{-i-i t}{\left(i+2 e_{1}\right)\left(i+(1-t) e_{1}\right)} .
\end{aligned}
$$

Hence $-f_{2}-f_{4} \leq 0$ whenever $\left(t, e_{1}\right) \in[0,1] \times[0, \infty)$. Combining these two observations, we have that:

$$
-\frac{\partial \log \mathfrak{c}_{j}}{\partial e_{1}}=-f_{1}-f_{2}-f_{3}-f_{4} \leq 0
$$

on the domain $[0,1] \times[0, \infty)$. A minimum of the function $\log \mathfrak{c}_{j}$ on the domain $[0,1] \times[0, \infty)$ thus occurs when $e_{1}=0$, and it follows that the same statement holds for the function $\mathfrak{c}_{j}$. Direct computation yields that $\mathfrak{c}_{j}(t, 0)=\left(\begin{array}{l}s \\ j\end{array}\right)$, which completes the proof when $j \notin\{1, s\}$.

If $j=1$, then we may still apply Lemma 3.3 and reduce to the case that $e_{i}=0$ for $i \neq 1$. Then we have:

$$
\mathfrak{b}_{1}\left(e_{1}\right)=\frac{\left(2+e_{1}\right)\left(3+e_{1}\right) \cdots\left(s+e_{1}\right)}{(s-1) !}
$$

which is at least than $\left(\begin{array}{l}s \\ 1\end{array}\right)$ whenever $e_{1} \geq 0$. If $j=s$, we reduce to the case that $e_{s}=e_{1}$ and we have:

$$
\mathfrak{b}_{s}\left(e_{1}, e_{s}\right)=\frac{\left(1+e_{1}\right) \cdots\left(s-1+e_{1}\right)}{(s-1) !}
$$

which is at least $\left(\begin{array}{l}s \\ s\end{array}\right)$ whenever $e_{1} \geq 0$.

Corollary 3.5. Let $\mathbf{d} \in \mathbb{Z}^{s+1}$ such that $d_{0} \leq 0$ and such that $d_{s}-s \leq 2 d_{1}-2$. Then:

$$
\beta_{j}(\bar{\pi}(\mathbf{d})) \geq\left(\begin{array}{l}
s \\
j
\end{array}\right)
$$


Proof. Let $\mathbf{e}=\left(e_{1}, \ldots, e_{s}\right)$ where $e_{i}=d_{i}-d_{i-1}-1$, so that $\mathfrak{d}(\mathbf{e})=\mathbf{d}$. Since $d_{s}=d_{0}+s+\sum_{i=1}^{s} e_{i}$ and $d_{1}=d_{0}+e_{1}+1$, we have that:

$$
\sum_{i=2}^{s} e_{i}=\left(d_{s}-s\right)-d_{0}-e_{1} \leq\left(2 d_{1}-2\right)-d_{0}-e_{1}=d_{1}-1 \leq e_{1} .
$$

The corollary now follows from Lemmas 3.1 and 3.4.

\section{Proof of Theorem 1.2}

We now prove our main result.

Proof of Theorem 1.2. By Theorem 2.1, we may write the Betti diagram of $M$ as a positive rational sum of pure diagrams:

$$
\beta(M)=\sum_{c \leq s \leq p} \sum_{\substack{d \in \operatorname{deg}\left(\mathbb{Z}^{p+1}\right) \\ \tau_{s}(\underline{\mathbf{d}}) \leq \mathbf{d} \leq \tau_{s}(\overline{\mathbf{d}})}} a_{\mathbf{d}} \bar{\pi}(\mathbf{d})
$$

By linearity, it is sufficient to show that:

$$
\beta_{j}(\bar{\pi}(\mathbf{d})) \geq\left(\begin{array}{l}
c \\
j
\end{array}\right)
$$

for every pure diagram appearing with nonzero coefficient in (3) and for every $j \in$ $\{1, \ldots, c\}$. Let $\mathbf{d}=\left(d_{0}, \ldots, d_{s}\right)$ be a degree sequence corresponding to such a pure diagram, and let $\mathbf{e}=\left(e_{1}, \ldots, e_{s}\right)$ defined by $e_{i}:=d_{i}-d_{i-1}-1$. Since $\bar{\pi}(\mathbf{d})$ appears with positive coefficient in equation (3), it must contribute to the Betti diagram $\beta(M)$. It follows that $d_{0} \leq 0$ and that

$$
d_{s}-s \leq \operatorname{reg}(M) \leq 2 \underline{d}_{1}(M)-2 \leq 2 d_{1}-2 .
$$

Hence $\mathbf{d}$ satisfies the hypotheses of Corollary 3.5 , and $\beta_{j}(\bar{\pi}(\mathbf{d})) \geq\left(\begin{array}{l}s \\ j\end{array}\right)$. Since $s \geq c$, it follows that $\left(\begin{array}{l}s \\ j\end{array}\right) \geq\left(\begin{array}{l}c \\ j\end{array}\right)$, and we obtain inequality (4).

Remark 4.1. With more care, one could show that equality in Theorem 1.2 may only occur in cases where $\operatorname{codim}(M) \leq 2$ or where there exists $m \in \mathbb{N}$ such that $M \cong k^{m}$ as a graded $S$-module.

\section{Asymptotic Betti Numbers}

Let $I$ be an ideal generated in a single degree $\delta$. The regularity of $I^{t}$ becomes a linear function $\operatorname{reg}\left(I^{t}\right)=\delta t+b$ for $t \gg 0$ (c.f. [22, Thm 3.2], [19, Cor 3]). We define $b$ to be the asymptotic regularity defect of $I$. The following theorem gives lower bounds for the Betti numbers of $S / I^{t}$.

Theorem 5.1. Let $I$ be an ideal of codimension c generated in a single degree $\delta$ and with asymptotic regularity defect $b$. We have the following lower bound on the Betti numbers of $S / I^{t}$ :

$$
\beta_{j}\left(S / I^{t}\right) \geq \frac{(b !)^{2} \delta^{c-1}}{(j-1+b) !(c-j+b) !} t^{c-1}+O\left(t^{c-2}\right)
$$

for all $j=1, \ldots, c$ and for all $t \gg 0$. 
Proof. By Theorem 2.1 we may write $\beta\left(S / I^{t}\right)$ as a sum of pure diagrams as in equation (3). Let $\mathbf{d}=\left(d_{0}, \ldots, d_{s}\right)$ be some degree sequence such that $\bar{\pi}(\mathbf{d})$ appears with nonzero coefficient in this sum. The equality $\operatorname{codim}\left(I^{t}\right)=\operatorname{codim}(I)=c$, implies that $s \geq c$. Let $\mathbf{e}=\left(e_{1}, \ldots, e_{s}\right)$ defined by $e_{i}=d_{i}-d_{i-1}-1$. Since $I^{t}$ is generated in degree $t \delta$, we have $e_{1}=t \delta$. Let $t \gg 0$ so that $\operatorname{reg}\left(S / I^{t}\right)=t \delta+b$. Since $\operatorname{reg}\left(S / I^{t}\right)=\bar{d}_{p}\left(S / I^{t}\right)-p$ we have that $\sum_{i=2}^{s} e_{i} \leq b$.

It is sufficient to prove the lower bound for the Betti numbers of the pure diagram $\bar{\pi}(\mathbf{d})$. In fact, it is sufficient to prove the lower bound for the functions $\mathfrak{b}_{j}(\mathbf{e})$ under the constraints $e_{1}=t \delta$ and $\sum_{i=2}^{s} e_{i} \leq b$. Let $j \in\{1, \ldots, c\}$. By Lemma 3.3, we may assume that $e_{i}=0$ unless $i \in\{1, j, j+1\}$. Hence we reduce to the case that $e_{j}+e_{j+1} \leq b$. We now seek to compute $\mathfrak{b}_{j}$.

$$
\mathfrak{b}_{j}(\mathbf{e})=\frac{(1+t \delta)(2+t \delta) \cdots(j-1+t \delta)(j+1+t \delta+b) \cdots(s+t \delta+b)}{\left(j-1+e_{j}\right) \cdots\left(1+e_{j}\right)\left(1+e_{j+1}\right) \cdots\left(s-j+e_{j+1}\right)}
$$

Note that $e_{j}$ and $e_{j+1}$ only appear in the denominator, and both are positive numbers less than $b$. Hence setting $e_{j}=e_{j+1}=b$ only decreases the right-hand side. This yields:

$$
\mathfrak{b}_{j}(\mathbf{e}) \geq \frac{(1+t \delta)(2+t \delta) \cdots(j-1+t \delta)(j+1+t \delta+b) \cdots(s+t \delta+b)}{(j-1+b) \cdots(1+b)(1+b) \cdots(s-j+b)}
$$

Since $s \geq c$ we may rewrite the right-hand side of (5) as

$$
\left(\frac{(1+t \delta)(2+t \delta) \cdots(j-1+t \delta)(j+1+t \delta+b) \cdots(c+t \delta+b)}{(1+b) \cdots(j-1+b)(1+b) \cdots(c-j+b)}\right)\left(\prod_{i=1}^{s-c} \frac{(c+i+t \delta+b)}{(c+i-j+b)}\right) .
$$

Each term of the product on the right is greater than 1, so by deleting this product and substituting back into (5), we obtain the inequality:

$$
\begin{aligned}
\mathfrak{b}_{j}(\mathbf{e}) & \geq \frac{(1+t \delta)(2+t \delta) \cdots(j-1+t \delta)(j+1+t \delta+b) \cdots(c+t \delta+b)}{(1+b) \cdots(j-1+b)(1+b) \cdots(c-j+b)} \\
& =\frac{(b !)^{2} \delta^{c-1}}{(j-1+b) !(c-j+b) !} t^{c-1}+O\left(t^{c-2}\right) .
\end{aligned}
$$

This completes the proof.

\section{Examples}

In this section, we consider several applications of Theorem 1.2, and we remark on the necessity of the hypothesis that $\operatorname{reg}(M) \leq 2 \underline{d}_{1}(M)-2$.

Example 6.1. Let $V \subseteq S_{d}$ be any vector space of forms of degree $d$ with $d>1$, and let $I \subseteq S$ be the ideal $V+\mathfrak{m}^{d+1}$. Then $S / I$ satisfies the hypotheses of Theorem 1.2. More generally, if $e \leq 2 d-1$ and $J$ is the ideal generated by $V$ and by $\mathfrak{m}^{e}$, then $S / J$ satisfies the hypotheses of Theorem 1.2.

Example 6.2 (Curves of High Degree). Let $C \subseteq \mathbb{P}^{n}$ be a smooth curve of genus embedded by a complete linear system of degree at least $2 g+1$. Let $I_{C} \subseteq k\left[x_{0}, \ldots, x_{n}\right]$ be the ideal defining $C$. Then $\operatorname{reg}\left(S / I_{C}\right) \leq 2$ by [12, Corollary 8.2]. Hence $S / I_{C}$ satisfies the hypotheses of Theorem 1.2, and thus $\beta_{i}\left(S / I_{C}\right) \geq\left(\begin{array}{c}n-1 \\ i\end{array}\right)$. 
Example 6.3 (Toric Surfaces). Let $X \subseteq \mathbb{P}^{n}$ be a toric surface embedded by a complete linear system $|A|$. Let $I_{X} \subseteq S=k\left[x_{0}, \ldots, x_{n}\right]$ be the defining ideal of $X$. We claim that $S / I_{X}$ satisfies the hypotheses of Theorem 1.2, and hence that $\beta_{i}\left(S / I_{X}\right) \geq\left(\begin{array}{c}n-2 \\ i\end{array}\right)$. Since $I_{X}$ has no generators in degree 1 , this amounts to showing that $\operatorname{reg}\left(S / I_{X}\right) \leq 2$. It is equivalent to show that the sheaf $\mathcal{I}_{X}:=\widetilde{I_{X}}$ is 3-regular [12, Prop 4.16].

We first check that $H^{1}\left(\mathbb{P}^{n}, \mathcal{I}_{X}(2)\right)=0$. Since $X$ is a toric surface and $A$ is an ample divisor, it follows from, for instance [21, Cor 2.1], that $X$ satisfies condition $N_{0}$, and hence that $X$ is projectively normal. The surjectivity of the map

$$
H^{0}\left(\mathbb{P}^{n}, \mathcal{O}_{\mathbb{P}^{n}}(2)\right) \rightarrow H^{0}\left(X, \mathcal{O}_{X}(2)\right)
$$

and the vanishing of $H^{1}\left(\mathbb{P}^{n}, \mathcal{O}_{\mathbb{P}^{n}}(2)\right)$ then imply that $H^{1}\left(\mathbb{P}^{n}, \mathcal{I}_{X}(2)\right)=0$.

We next check that $H^{2}\left(\mathbb{P}^{n}, \mathcal{I}_{X}(1)\right)=0$. This follows from the exact sequence:

$$
H^{1}\left(X, \mathcal{O}_{X}(1)\right) \rightarrow H^{2}\left(\mathbb{P}^{n}, \mathcal{I}_{X}(1)\right) \rightarrow H^{2}\left(\mathbb{P}^{n}, \mathcal{O}_{\mathbb{P}^{n}}(1)\right)
$$

and the fact that higher cohomology of ample line bundles vanishes on toric varieties. $W e$ conclude that $\mathcal{I}_{X}$ is 3-regular, which implies that $S / I_{X}$ satisfies the hypotheses of Theorem 1.2.

Example 6.4. Let $I$ be any ideal with minimal degree generator in degree $\underline{d}_{1}$ and maximal degree generator in degree $\bar{d}_{1}$. Assume that $\bar{d}_{1}(I)<2 \underline{d}_{1}(I)$. Then

$$
\operatorname{reg}\left(S / I^{t}\right) \leq t \bar{d}_{1}+b
$$

for some $b$ and for all $t \geq 1\left[10\right.$, Thm 1.1(i)]. Since $\bar{d}_{1}(I)<2 \underline{d}_{1}(I)$, it follows that, for all $t \gg 0, t \bar{d}_{1}(I)+b<2 t \underline{d}_{1}(I)-2$. Hence, for all $t \gg 0$, the module $S / I^{t}$ satisfies the hyoptheses of Theorem 1.2.

The method of proof for Theorem 1.2 breaks down if one removes the hypothesis that $\operatorname{reg}(M) \leq 2 \underline{d}_{1}(M)-2$. One issue is that the statement:

$$
\beta_{j}(M) \geq \beta_{0}(M)\left(\begin{array}{c}
\operatorname{codim}(M) \\
j
\end{array}\right)
$$

is not true in general. For example, if $S=k[x, y]$ and $N$ is the cokernel of a generic $2 \times 3$ matrix of linear forms, then

$$
\beta_{1}(N)=3<4=\beta_{0}(N)\left(\begin{array}{c}
\operatorname{codim}(N) \\
1
\end{array}\right) .
$$

There also exist pure diagrams with integral entries which do not satisfy the graded BEH rank conjecture. For instance, the diagram:

$$
\bar{\pi}(0,1,2,3,5,6)=\left(\begin{array}{cccccc}
1 & \frac{9}{2} & \frac{15}{2} & 5 & - & - \\
- & - & - & - & \frac{3}{2} & \frac{1}{2}
\end{array}\right)
$$

does not satisfy any version of Conjecture 1.1.

\section{Acknowledgements}

We thank Kiril Datchev for helpful discussions about the proof of Lemma 3.4, David Eisenbud for his guidance, Milena Hering for suggesting improvements to Example 6.3, and Tony Várilly-Alvarado for comments on an early draft. 


\section{References}

[1] M. Boij and J. Söderberg, Betti numbers of graded modules and the Multiplicity Conjecture in the non-Cohen-Macaulay case, arXiv (2008), no. 0803.1645, 1-14.

[2] - Graded Betti numbers of Cohen-Macaulay modules and the multiplicity conjecture, J. Lond. Math. Soc. (2) 78 (2008), no. 1, 85-106.

[3] D. A. Buchsbaum and D. Eisenbud, Algebra structures for finite free resolutions, and some structure theorems for ideals of codimension 3, Amer. J. Math. 99 (1977), no. 3, 447-485.

[4] G. Carlsson, Free $(\mathbf{Z} / 2)^{k}$-actions and a problem in commutative algebra, in Transformation groups, Poznań 1985, Vol. 1217 of Lecture Notes in Math., 79-83, Springer, Berlin (1986).

[5] S.-T. Chang, Betti numbers of modules of exponent two over regular local rings, J. Algebra 193 (1997), no. 2, 640-659.

[6] - Betti numbers of modules of essentially monomial type, Proc. Amer. Math. Soc. 128 (2000), no. 7, 1917-1926.

[7] H. Charalambous, Betti numbers of multigraded modules, J. Algebra 137 (1991), no. 2, 491-500.

[8] H. Charalambous, E. G. Evans, and M. Miller, Betti numbers for modules of finite length, Proc. Amer. Math. Soc. 109 (1990), no. 1, 63-70.

[9] H. Charalambous and E. G. Evans, Jr., Problems on Betti numbers of finite length modules, in Free resolutions in commutative algebra and algebraic geometry (Sundance, UT, 1990), Vol. 2 of Res. Notes Math., 25-33, Jones and Bartlett, Boston, MA (1992).

[10] S. D. Cutkosky, J. Herzog, and N. V. Trung, Asymptotic behaviour of the Castelnuovo-Mumford regularity, Compositio Math. 118 (1999), no. 3, 243-261.

[11] D. Dugger, Betti numbers of almost complete intersections, Illinois J. Math. 44 (2000), no. 3, 531-541.

[12] D. Eisenbud, The geometry of syzygies, Vol. 229 of Graduate Texts in Mathematics, SpringerVerlag, New York (2005), ISBN 0-387-22215-4. A second course in commutative algebra and algebraic geometry.

[13] D. Eisenbud and F.-O. Schreyer, Betti Numbers of graded modules and cohomology of vector bundles, arXiv (2007), no. 0712.1843, 1-42.

[14] E. G. Evans and P. Griffith, Binomial behavior of Betti numbers for modules of finite length, Pacific J. Math. 133 (1988), no. 2, 267-276.

[15] R. Hartshorne, Algebraic vector bundles on projective spaces: a problem list, Topology 18 (1979), no. 2, 117-128.

[16] J. Herzog and M. Kühl, On the Betti numbers of finite pure and linear resolutions, Comm. Algebra 12 (1984), no. 13-14, 1627-1646.

[17] M. Hochster and B. Richert, Lower bounds for Betti numbers of special extensions, J. Pure Appl. Algebra 201 (2005), no. 1-3, 328-339.

[18] C. Huneke and B. Ulrich, The structure of linkage, Ann. of Math. (2) 126 (1987), no. 2, 277-334.

[19] V. Kodiyalam, Asymptotic behaviour of Castelnuovo-Mumford regularity, Proc. Amer. Math. Soc. 128 (2000), no. 2, 407-411.

[20] L. Santoni, Horrocks' question for monomially graded modules, Pacific J. Math. 141 (1990), no. 1, 105-124.

[21] H. Schenck, Lattice polygons and Green's theorem, Proc. Amer. Math. Soc. 132 (2004), no. 12, 3509-3512 (electronic).

[22] N. V. Trung and H.-J. Wang, On the asymptotic linearity of Castelnuovo-Mumford regularity, J. Pure Appl. Algebra 201 (2005), no. 1-3, 42-48.

Department of Mathematics, University of California, Berkeley, CA 94720-3840, USA

E-mail address: derman@math.berkeley.edu

$U R L:$ http://math.berkeley.edu/ derman 